Journal for ImmunoTherapy of Cancer

\section{Bone marrow MSC from pediatric patients with B-ALL highly immunosuppress $T$-cell responses but do not compromise CD19-CAR T- cell activity}

To cite: Zanetti SR, Romecin PA, Vinyoles $\mathrm{M}$, et al. Bone marrow MSC from pediatric patients with B-ALL highly immunosuppress T-cell responses but do not compromise CD19-CAR T-cell activity. Journal for ImmunoTherapy of Cancer 2020;8:e01419. doi:10.1136/ jitc-2020-001419

SRZ and PAR contributed equally.

Accepted 08 August 2020

Check for updates

(c) Author(s) (or their employer(s)) 2020. Re-use permitted under CC BY-NC. No commercial re-use. See rights and permissions. Published by BMJ.

For numbered affiliations see end of article.

\section{Correspondence to}

Dr Samanta Romina Zanetti; szanetti@carrerasresearch.org

Dr Pablo Menendez; pmenendez@carrerasresearch. org

\section{ABSTRACT}

Background Although adoptive transfer of CD19-directed chimeric antigen receptor (CAR) T-cells (CD19-CAR T-cells) achieves high rates of complete response in patients with B-cell acute lymphoblastic leukemia (B-ALL), relapse is common. Bone marrow (BM) mesenchymal stem/stromal cells (BM-MSC) are key components of the hematopoietic niche and are implicated in B-ALL pathogenesis and therapy resistance. MSC exert an immunosuppressive effect on T-cells; however, their impact on CD19-CAR Tcell activity is understudied.

Methods We performed a detailed characterization of BM-MSC from pediatric patients with B-ALL (B-ALL BM$M S C)$, evaluated their immunomodulatory properties and their impact on CD19-CAR T-cell activity in vitro using microscopy, qRT-PCR, ELISA, flow cytometry analysis and in vivo using a preclinical model of severe colitis and a B-ALL xenograft model.

Results While B-ALL BM-MSC were less proliferative than those from age-matched healthy donors (HD), the morphology, immunophenotype, differentiation potential and chemoprotection was very similar. Likewise, both BMMSC populations were equally immunosuppressive in vitro and anti-inflammatory in an in vivo model of severe colitis. Interestingly, BM-MSC failed to impair CD19-CAR T-cell cytotoxicity or cytokine production in vitro using B-ALL cell lines and primary B-ALL cells. Finally, the growth of NALM6 cells was controlled in vivo by CD19-CAR T-cells irrespective of the absence/presence of BM-MSC. Conclusions Collectively, our data demonstrate that pediatric B-ALL and HD BM-MSC equally immunosuppress T-cell responses but do not compromise CD19-CAR T-cell activity.

\section{BACKGROUND}

Acute lymphoblastic leukemia (ALL) is the most common malignancy in childhood, accounting for $\sim 25 \%$ of all childhood cancers. ${ }^{1}$ B-cell ALL (B-ALL) represents $~ 80 \%$ of ALL and is an aggressive hematologic malignancy characterized by the clonal expansion of
CD $19^{+}$B-cell precursors. ${ }^{1}$ Of special interest is the B-ALL harboring chromosomal rearrangements of the mixed-lineage leukemia gene (MLL-r), which presents unique clinical and biological features and has a dismal prognosis compared with other B-ALL. ${ }^{2}$

B-ALL originates in the bone marrow $(\mathrm{BM}){ }^{3}$ and the $\mathrm{BM}$ microenvironment (BMM) is particularly important in the pathogenesis of the B-ALL given the high dependence of leukemic cells on external factors for proliferation and survival. ${ }^{4}$ The $\mathrm{BM}$ is also the primary site where residual leukemic cells survive during standard chemotherapy, ${ }^{5} 6$ and is the most frequent location of B-ALL relapse. ${ }^{7}$ Mesenchymal stem/stromal cells (MSC) are major components of the BMM and are involved in the pathogenesis and drug resistance of B-ALL cells during the chemotherapy, ${ }^{8-14}$ underscoring the active investigations aimed at unraveling the contribution of BM-MSC to B-ALL cell response to treatment.

Adoptive transfer of T-cells engineered to express an artificial chimeric antigen receptor (CAR) targeting a tumor cell surface-specific antigen is a promising new approach for cancer immunotherapy. In the setting of B-ALL, CAR T-cells targeting CD19 have generated unprecedented results in multiple clinical trials, and $\sim 50 \%$ of treated patients are disease-free after 12 months. ${ }^{15} 16$ Despite these robust clinical responses, however, leukemia relapse after treatment is common and remains a major challenge for CD19-CAR T-cell therapy. ${ }^{17}$ An intriguing result of CD19-CAR T-cell therapy in B-ALL is that MLL-r B-ALL patients show lower response rates and higher rates of 
immune escape. ${ }^{18}$ The antitumor efficacy and persistence of CD19-CAR T-cells within the host depend on complex in vivo processes and multicellular interactions and, in recent years, it has been extensively demonstrated that MSC regulate T-cells by modifying their activation, proliferation and effector functions. ${ }^{19} 20$ To the best of our knowledge, the immunomodulatory properties of pediatric B-ALL-derived BM-MSC in vivo, and their impact on CAR T-cell efficacy/resistance in B-ALL remains unexplored. Thus, in the present study, we sought to characterize in vitro and in vivo the immunomodulatory effects of B-ALL BM-MSC on T-cells and CD19-CAR T-cells, and compared them with healthy donors (HD) BM-MSC. A better understanding of the interactions between T-cells and the B-ALL BMM will help improving the efficiency of current immunotherapies in B-ALL.

\section{MATERIALS AND METHODS}

Reagents, drugs, antibodies and cell lines

Advanced DMEM, IMDM, RPMI-1640, L-glutamine and penicillin-streptomycin $(\mathrm{P} / \mathrm{S})$, insulin-transferrinselenium (ITS) were purchased from Gibco/Invitrogen, Waltham, Massachusetts, USA. StemSpan SFEM was purchased from STEMCELL Technologies StemCell Technologies, Vancouver, Canada. Phosphate buffered saline was purchased from Merck Life Science SL, Madrid, Spain. MSC osteogenic and adipogenic differentiation medium were purchased from Promocell, Heidelberg, Alemania. Human (h) Stem Cell Factor (SCF), hFMS-like tyrosine kinase 3 ligand (FLT3-L), human interleukin-3 (hIL-3), hIL-7 and hIL-15 were purchased from Miltenyi Biotec, Bergisch Gladbach, Germany. Nitro blue tetrazolium chloride (NBT), alizarin red, oil red-O, dexamethasone, L-phytohemagglutinin (PHA-L) and fetal bovine serum (FBS) were purchased from Sigma-Aldrich, St. Louis, Missouri, USA. L-asparaginase was purchased from Jazz Pharmaceuticals Iberia SL, Barcelona, Spain. CellTrace Violet Cell Proliferation Kit and CellTrace CFSE Cell Proliferation Kit were purchased from Fisher Scientific SL, Madrid, Spain. Cell fixation and permeabilization Kit was purchased from Nordic-Mubio, Derio, Spain.
Anti-CD3 (OKT3) and anti-CD28 (CD28.2) monoclonal antibodies (mAbs), 7-amino-actinomycin D (7-AAD), phycoerythrin (PE) annexin $\mathrm{V}$ apoptosis detection kit and fluorescein isothiocyanate, $\mathrm{PE}$, peridin chlorophyll, allophycocyanin (APC)-, PE/ cyanine7 (PE/Cy7)-, brilliant violet 421 (BV421), BV510)-conjugated mAbs specific for human CD3, CD19, CD45, CD13, CD73, CD105, CD90, CD31, CD34 and isotype-matched negative control mAbs were purchased from BD Bioscience, Franklin Lakes, New Jersey, USA. APC-conjugated anti-Nestin mAb was purchased from R\&D System, Abingdon, UK. The B-ALL cell lines SEM and NALM6 were obtained from DSMZ cell line bank, Braunschweig, Germany. Luciferase (Luc)/ GFP-expressing-NALM6 cells were kindly provided by RJ Brentjens (MSKCC, New York, USA).

\section{Human samples}

Peripheral blood (PB) mononuclear cells (PBMC) were isolated from buffy coats of healthy volunteers by Ficoll-Hypaque gradient centrifugation (GE Healthcare, Chicago, Illinois, USA) ${ }^{21}$ Buffy coats were obtained from the Catalan Blood and Tissue Bank (BST) on Institutional Review Board approval (HCB/2018/0030). BM B-ALL aspirates were obtained from pediatric patients with ETV6/RUNX1 or MLL-r B-ALL (table 1). BM aspirates from age-matched HD were obtained from the BST. Human samples were obtained after written informed consent in accordance with the Declaration of Helsinki.

\section{Isolation, expansion and characterization of BM-MSC}

Mononuclear cells (MC) from BM samples were isolated by centrifugation on Ficoll-Paque Plus density gradient. MCs were seeded at $2 \times 10^{5}$ cells $/ \mathrm{cm}^{2}$ in advanced DMEM with $10 \%$ heat-inactivated FBS, L-glutamine and $\mathrm{P} / \mathrm{S}$ (complete advanced DMEM medium) and maintained in a humidified atmosphere with $5 \% \mathrm{CO}_{2}$ at $37^{\circ} \mathrm{C} 2$ days, after which non-adherent cells were collected and frozen and fresh medium was added. Adherent cells at $>85 \%$ confluence were trypsinized and replated at $5 \times 10^{3}$ cells $/ \mathrm{cm}^{2}{ }^{22}$ The resulting BM-MSC cultures were maintained in complete Advanced DMEM medium. BM-MSC cultures were assessed daily for changes in growth rate

Table 1 Biological and molecular characteristics of HD and B-ALL patients

\begin{tabular}{lllllll}
\hline HD/patient & Disease stage & Cytogenetic & Molecular & Age (years) & Gender & Blasts (\%) \\
\hline HD \#1 & N.A. & N.A. & N.A. & 9 & Female & N.A. \\
HD \#2 & N.A. & N.A. & N.A. & 10 & Female & N.A. \\
HD \#3 & N.A. & N.A. & N.A. & 7 & Male & N.A. \\
$\mathrm{Pt} \mathrm{\# 1}$ & Diagnosis & $\mathrm{t}(12 ; 21)$ & ETV6/RUNX1 & 5 & Male & 84 \\
$\mathrm{Pt} \mathrm{\# 2}$ & Diagnosis & $\mathrm{t}(12 ; 21)$ & ETV6/RUNX1 & 2 & Male & 80 \\
$\mathrm{Pt} \mathrm{\# 3}$ & Diagnosis & $\mathrm{t}(9 ; 11)$ & MLL/AF9 & 3 & Male & 18 \\
$\mathrm{Pt} \mathrm{\# 4}$ & Diagnosis & $\mathrm{t}(1 ; 11)$ & MLL/EPS15 & 1 & Female & 80 \\
$\mathrm{Pt} \mathrm{\# 5}$ & Relapse & $\mathrm{t}(4 ; 11)$ & MLL/AF4 & 4 & Female & 81 \\
\hline
\end{tabular}

B-ALL, B-cell acute lymphoblastic leukemia; HD, healthy donors; N.A, not aplicable. 
and morphology. All experiments were performed with cells harvested between passage (P) 2 and $\mathrm{P} 5$.

Growth kinetics of BM-MSC were measured as cumulative Population Doublings at each passage, as described. ${ }^{23}$ An equal number of BM-MSC $\left(5 \times 10^{3}\right.$ cells $\left./ \mathrm{cm}^{2}\right)$ at P1 were initially plated for each patient/donor; cells were then harvested every 5 days, stained with trypan blue, counted and re-plated at $5 \times 10^{3}$ cells $/ \mathrm{cm}^{2}$. The procedure was repeated until $\mathrm{P} 6$.

BM-MSC characterization was performed following the guidelines proposed by the International Society for Cellular Therapy (ISCT). ${ }^{24}$ The immunophenotype of cultured BM-MSC was assessed by FACS. A total of $1 \times 10^{6}$ cells were incubated for $30 \mathrm{~min}$ at $4^{\circ} \mathrm{C}$ in the dark. Data acquisition and analysis were performed using a FACSCanto-II flow cytometer equipped with FACSDiva software (BD Biosciences). Differentiation was evaluated by plating BM-MSC in specific differentiation induction medium for 3-4 weeks. For osteogenic differentiation, BM-MSCs were stained to determine alkaline phosphatase (AP) activity using NBT and for calcium deposits with alizarin red. $^{25}$ For adipogenic differentiation, BM-MSCs were stained with oil red-O to detect the lipid droplets. ${ }^{22}$ Differentiation capacity was further quantified by quantitative RT-PCR (qRT-PCR) and RNA was isolated from differentiated BM-MSC cultures using the Maxwell RSC simplyRNA Cells Kit (Promega, Madison, Wisconsin, USA). RT-PCR analysis was conducted using the SYBR Green PCR Master Mix (Fisher Scientific SL) with primers for specific adipogenic differentiation and osteogenic differentiation transcription factors. Analysis and mRNA quantification analysis were performed on the CFX384 Real-Time System (Bio-Rad Laboratorie, Hercules, California, USA). Reactions were performed in triplicate and gene expression values were normalized to those of HPRT. Gene-specific primer sequences for qRTPCR are listed in online supplementary Table S1.

\section{T-cell proliferation and cytokine secretion}

HD and B-ALL BM-MSC were seeded at $1 \times 10^{4}$ cells / well in flat-bottom 96-well plates containing complete Advanced DMEM medium at $37^{\circ} \mathrm{C}$ for 24 hours. The next day, we evaluated T-cell proliferation using the CellTrace CFSE Cell Proliferation Kit. To do this, PBMCs were labeled with $5 \mu \mathrm{M}$ CellTrace CFSE for $20 \mathrm{~min}$ at $37^{\circ} \mathrm{C}$ and then washed three times with RPMI 1640/1\% heat-inactivated $\mathrm{FBS}^{26}$ Subsequently, the BM-MSC culture medium was removed and CellTrace CFSE-labeled cells were added to the adherent BM-MSC culture at a BM-MSC:PBMC ratio of 1:5 and 1:10 in RPMI-1640 with10\% heat-inactivated FBS and P/S (complete RPMI medium) (or plated alone), in the absence or presence of PHA-L $(1 \mu \mathrm{g} / \mathrm{mL})$. After 3 and 6 days of culture, non-adherent cells were collected, washed and stained with an anti-CD3 mAb and 7-AAD. Viable cells were gated as 7-AAD'. Cell division was measured using dye dilution on the $\mathrm{CD}^{+}$population by FACS and the number of proliferating cells was determined by gating on the $\mathrm{CD}^{+} \mathrm{CFSE}^{\text {low }}$ subset. ${ }^{26}$
Quantification of the proinflammatory cytokines IL-2, interferon- $\gamma($ IFN- $\gamma$ ) and tumor necrosis factor $\alpha$ (TNF- $\alpha$ ) was measured by ELISA using the OptEIA Human ELISA Kit (BD Biosciences) on supernatants from the aforementioned BM-MSC:PBMC cocultures. Unstimulated PBMC:BM-MSC cocultures were processed in parallel as a control. ELISA determinations were performed in triplicate.

\section{In vitro chemoresistance of B-ALL cell lines}

HD and B-ALL BM-MSC were seeded at $1 \times 10^{4}$ cells/well in flat-bottom 96-well plates containing complete Advanced DMEM medium at $37^{\circ} \mathrm{C}$ for 24 hours. The next day, the BM-MSC culture medium was removed and SEM cells $\left(1 \times 10^{5}\right.$ cells $/$ well $)$ were added to the adherent BM-MSC culture at a BM-MSC:SEM ratio of 1:10 in IMDM/10\% heat-inactivated FBS and $\mathrm{P} / \mathrm{S}$, in the absence or presence of dexamethasone (10 UI) or L-asparaginase $(1 \mu \mathrm{M})$. Two days after, non-adherent cells were collected, washed and stained with an anti-CD19 mAb, 7-AAD and AnnexinV to determine cell apoptosis by FACS. ${ }^{27}$

\section{CD19-CAR vector, lentiviral production, T-cell transduction, activation and expansion}

We used our clinically validated pCCL second-generation lentiviral CD19-CAR backbone, which contains a human CD8 transmembrane domain and human 4-1BB and CD3 $\zeta$ endodomains. ${ }^{28}$ We incorporated a GFP reporter gene after a $2 \mathrm{~A}$ ribosomal skip sequence at the $\mathrm{C}$-terminal CAR sequence to evaluate the transduction efficiency and the tracking of CAR expression. CAR-expressing viral particles pseudotyped with VSV-G were generated by transfection of HEK $293 \mathrm{~T}$ cells with pCCL, VSV-G and psPAX2 vectors using polyethylenimine (Polysciences, Warrington, Pennsylvania, USA). Supernatants were collected at 48 and 72 hours after transfection and concentrated by ultracentrifugation.

T-cells were activated by plate-coating with anti-CD3 and anti-CD28 mAbs in complete RPMI medium for 2 days and were then transduced with a CAR-expressing lentivirus at a multiplicity of infection of 10 in the presence of hIL-7 and hIL-15 $(10 \mathrm{ng} / \mathrm{mL}) .{ }^{29}$ T-cells were expanded in complete RPMI medium plus hIL-7 and hIL-15 for up to 6 days. CAR transduction efficiency in T-cells was analyzed by FACS.

\section{In vitro CD19-CAR T-cell, cytotoxicity assays and cytokine release determination}

For cytotoxicity assays, BM-MSCs were seeded at $1 \times 10^{4}$ cells/well in flat-bottom 96-well plates containing complete Advanced DMEM medium and incubated at $37^{\circ} \mathrm{C}$ for 24 hours. The next day, target cells (B-ALL cell lines or primary B-ALL cells, $1 \times 10^{5}$ target cells/well) were incubated with CD19-CAR T-cells at an Effector:Target (E:T) ratio of 1:1, or alone, for the indicated periods in the absence/presence of BM-MSC. Cell lines were cultured in RPMI complete medium and primary cells were cultured in StemSpan supplemented with $20 \%$ heat-inactivated 
FBS, P/S, ITS, hSCF (100 ng/mL), hFLT3-L (100 ng/ $\mathrm{mL})$, hIL-3 $(10 \mathrm{ng} / \mathrm{mL})$ and hIL-7 $(10 \mathrm{ng} / \mathrm{mL})$. After 2 and 6 days of culture, non-adherent cells were collected, washed and stained with anti-CD3, anti-CD19, anti-CD10, anti-CD22 mAbs and 7-AAD. CAR T-cell-mediated cytotoxicity was determined by analyzing the residual living $\left(\mathrm{CD} 10^{+} \mathrm{CD} 22^{+} 7-\mathrm{AAD}^{-}\right)$target cells at each time point. BD TruCount absolute count tubes (BD Biosciences) were used for absolute cell counting. Quantification of the pro-inflammatory cytokines IL-2, IFN- $\gamma$ and TNF- $\alpha$ was measured by ELISA using the OptEIA Human ELISA Kit (BD Biosciences) on supernatants harvested after 2 days of coculture at a 1:1 E:T ratio. ELISA determinations were performed in triplicate.

\section{Induction and treatment of experimental acute severe colitis}

A well-established experimental mouse model for bowel inflammatory disease/acute colitis was employed.$^{3031}$ The in vivo procedure was approved by the Ethics Committee of the Spanish Council of Scientific Research. To induce acute colitis, 2,4,6-trinitrobenzene sulfonic acid (TNBS, 2mg; Sigma) in 50\% ethanol $(100 \mu \mathrm{L})$ was administered intrarectally in male BALB/c mice aged 6-8weeks ( $\mathrm{n}=10 /$ group). Control mice received $50 \%$ ethanol alone. At 4 hours after TNBS infusion, animals were treated intraperitoneally with medium alone, with $1 \times 10^{6} \mathrm{HD}$ BM-MSCs $(\mathrm{n}=3)$ or with $1 \times 10^{6}$ B-ALL BM-MSC (MLL-r B-ALL $\mathrm{n}=3$ ), resuspended in $200 \mu \mathrm{l}$ of medium. Animals were monitored daily for the appearance of diarrhea, body weight loss, and survival. Colitis signs were scored based on stool consistency and rectal bleeding by a blinded observer using the following scale: $0=$ normal stool appearance, $1=$ slight decrease in stool consistency, $2=$ moderate decrease in stool consistency, $3=$ moderate decrease in stool consistency and presence of blood in stools, $4=$ severe watery diarrhea and moderate/severe blooding in stools. At day 9 or immediately after the death of the animal, the colons were collected and evaluated for macroscopic damage (scale 0-8) based on the grade of tissue adhesion, presence of ulceration and wall thickness in a blinded fashion by a researcher: ulceration $(0=$ normal appearance, $1=$ focal hyperemia, no ulcers, $2=$ ulceration without hyperemia or bowel wall thickening, $3=$ ulceration with inflammation at 1 site, $4=$ two or more sites of ulceration and inflammation, $5=$ major sites of damage extending $>1 \mathrm{~cm}$ along length of colon), adhesions $(0=$ no adhesions, $1=$ minor adhesions, colon can be easily separated from the other tissues, $2=$ major adhesions); and thickness (maximal bowel wall thickness, in $\mathrm{mm}$, measured with a caliper). PB was collected from the tail vein (day 4) and by cardiac puncture (day 9) and the levels of proinflammatory cytokines were determined in sera by specific sandwich ELISA using capture/biotinylated detection antibodies from PreproTech.

\section{In vivo CD19-CAR $T$ cell-mediated cytotoxicity assay}

Ten-week-old non-obese diabetic Cg-Prkdc ${ }^{\text {scid }}$ Il2rg $^{\text {tm1wjl }}$ / SzJ (NSG) mice (The Jackson Laboratory, Bar Harbor,
Maine, USA) were bred and housed under pathogen-free conditions. NSG mice were intratibial (IT)-injected with $1 \times 10^{6}$ NALM6-Luc ${ }^{+}$cells alone or together with $3 \times 10^{5}$ BM-MSC, ${ }^{27}$ followed 4 days later by an intravenous infusion of $4 \times 10^{6}$ CD19-CAR T-cells. Mice were followed up weekly by bioluminescence (BLI) using an in vivo imaging system (Lumina III; Perkin-Elmer, Wlatham, Massachusetts, USA). Mice were sacrificed at day 15 (controls) and day 35 (CAR-treated), and PB and BM samples were collected and analyzed by FACS to assess leukemic burden and CAR T-cell persistence.

\section{Statistical analysis}

Data were plotted as mean \pm SEM. One-way analysis of variance with Tukey's post hoc test was used when three or more experimental groups were compared and Student's paired t-test was used when two experimental groups were compared. All analyzes were performed with Prism software, V.8.0 (GraphPad Prism Software, San Diego, California, USA).

\section{RESULTS}

\section{Expansion and characterization of bona fide BM-MSC from pediatric patients with B-ALL}

We isolated MSC from BM aspirates of 5 pediatric B-ALL, (2 ETV6/RUNX1 and 3 MLL-r), and we compared their morphology, immunophenotype, growth kinetics and differentiation potential with that of BM-MSC from three pediatric HD counterparts using the standardized criteria outlined by the ISCT. ${ }^{24}$ Table 1 shows the main biological and molecular/cytogenetic characteristics of B-ALL patients and HD. Adherent cell cultures were successfully established from all samples using standard MSC culture conditions. ${ }^{24}$ BM-MSC from both groups adhered to plastic and showed homogeneous spindle-shaped fibroblast-like morphology (figure 1A, online supplementary figure S1A). FACS analysis at P3 revealed that both BM-MSC groups display a bona fide MSC immunophenotype positive for CD105, CD90, CD73 and CD13 and Nestin, while negative for hematopoietic (CD34 and CD45) and endothelial (CD31) cell markers (figure 1B, online supplementary figure S1B) ${ }^{22}$ When we compared their expansion potential in vitro under identical culture conditions, HD BM-MSC showed a significantly superior proliferative potential than that of B-ALL BM-MSC (figure 1C). We next evaluated the differentiation potential of P3 BM-MSC cultures by exposing them to adipogenic and osteoblastic induction conditions. Both groups were similar with regard to adipogenic differentiation, measured qualitatively by the formation of lipid droplets detected by oil red-O staining; and to osteoblast differentiation, revealed by AP activity staining and by the presence of calcium deposits detected by Alizarin Red staining (figure 1D). Adipogenic and osteoblastic differentiation capacity was also confirmed quantitatively by qPCR, which showed a comparable and significant increase in the expression of early and late master regulator genes 


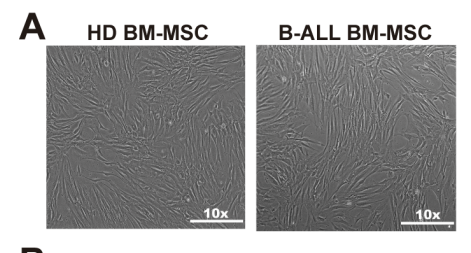

C

B
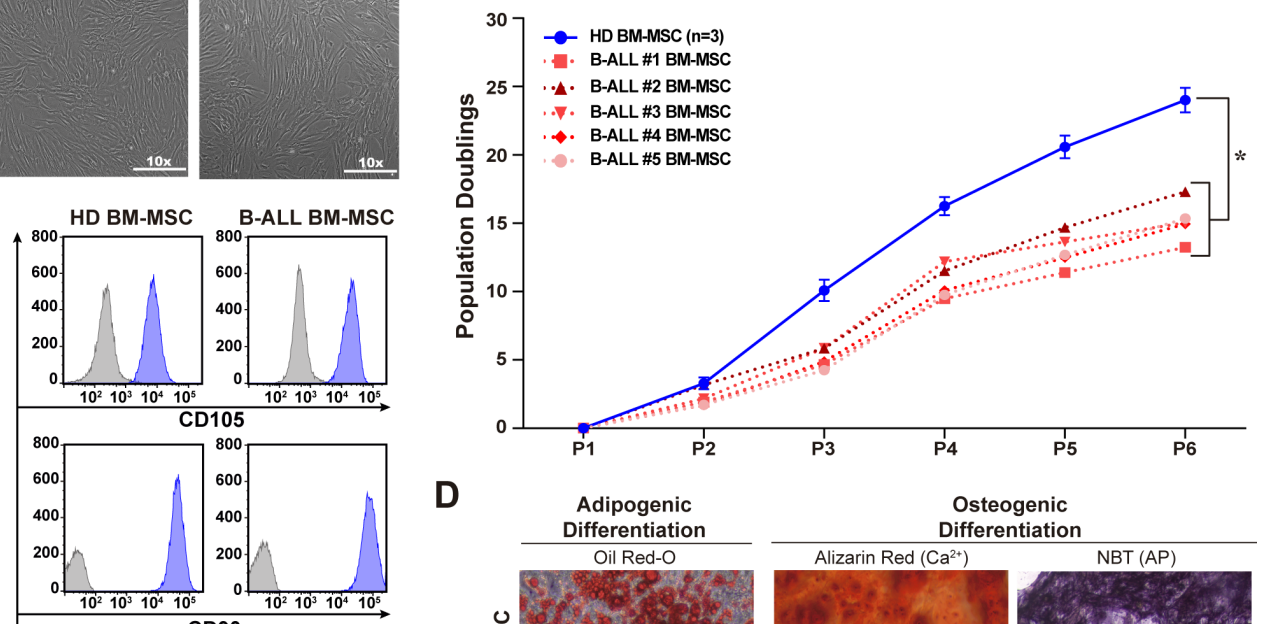

D
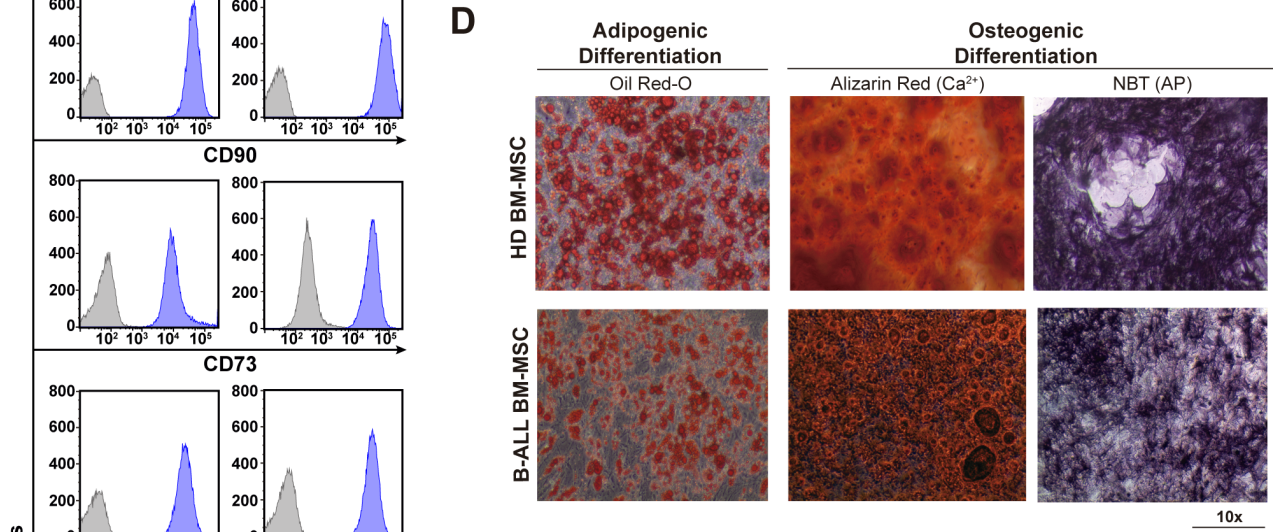

E
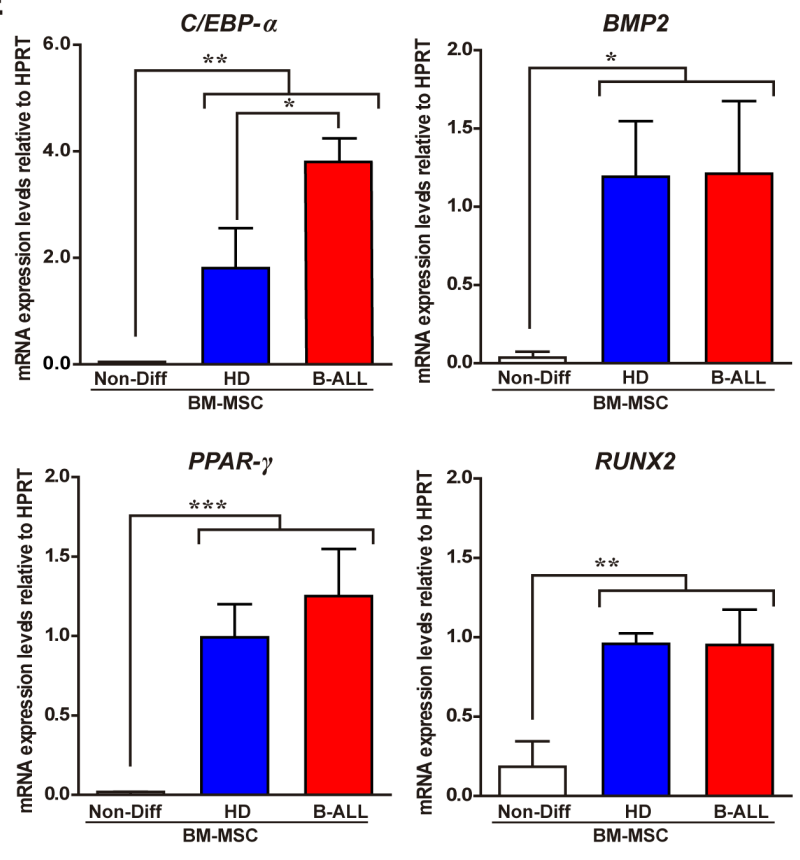

Figure 1 Characterization of BM-MSC from pediatric patients with B-ALL and age-matched HD. (A) Representative phasecontrast morphology of BM-MSC. (B) Immunophenotype of expanded BM-MSC at P3. Expression of CD105, CD90, CD73, CD13, nestin, CD45, CD34 and CD31 was analyzed by FACS. Shown are representative FACS histograms; gray histograms represent isotype-matched negative control $\mathrm{mAb}$ and blue histograms represent $\mathrm{mAb}$-specific stained cells. (C) In vitro proliferation of BM-MSC calculated as population doubling over six passages. (D, E) Osteogenic and adipogenic differentiation capacity of BM-MSC. (D) Left panel, oil red-O staining indicative of adipogenic differentiation capacity. Right panel, Alizarin red staining and detection of AP alkaline phosphatase activity with NTB, indicative of osteogenic differentiation capacity. (E) Relative quantification of mRNA expression of the adipogenic transcription factors $C / E B P-\alpha$ and $P P A R-\gamma$ and the osteogenic transcription factors BMP2 and RUNX2 by qRT-PCR. Data are shown as mean \pm SEM. ${ }^{*} P<0.05,{ }^{* *} p<0.01,{ }^{* \star *} \mathrm{p}<0.001$; one-way ANOVA with Tukey's post hoc test. HD BM-MSC $n=3$ and B-ALL BM-MSC $n=5$. ANOVA, analysis of variance; B-ALL, B-cell acute lymphoblastic leukemia; BM-MSC, bone marrow-mesenchymal stem/stromal cells; HD, healthy donors; NTB, nitro blue tetrazolium. 
A

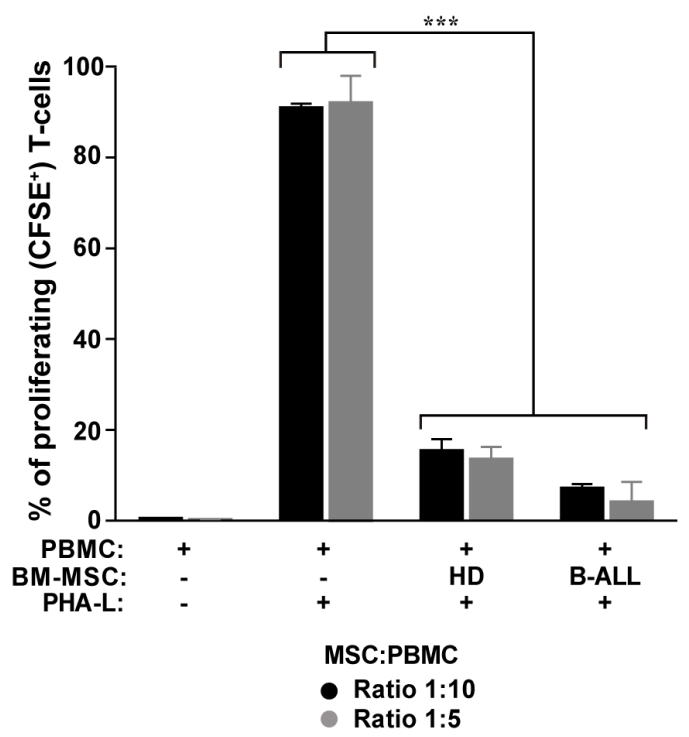

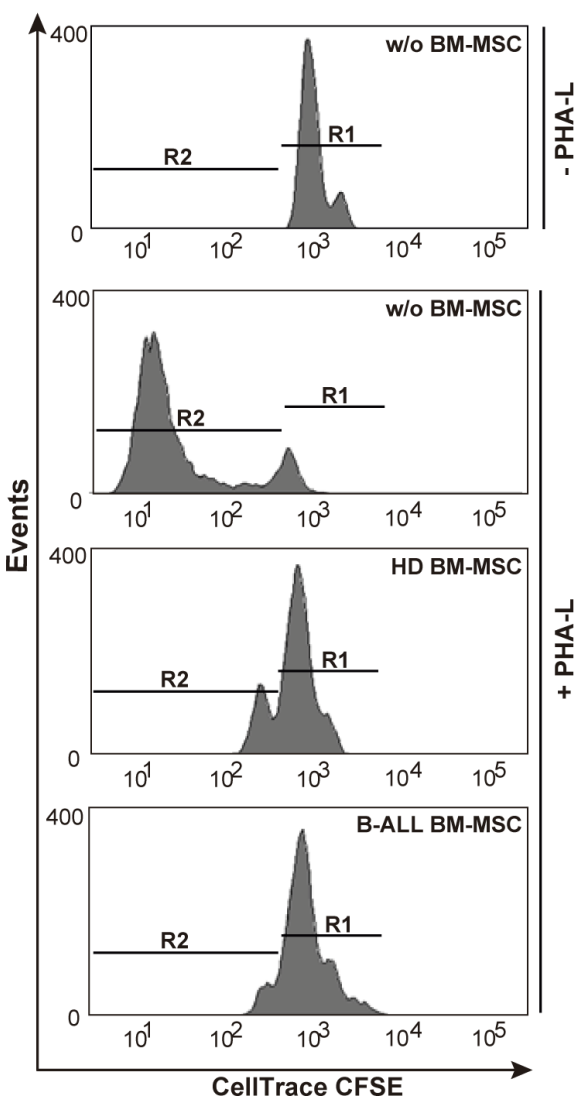

B
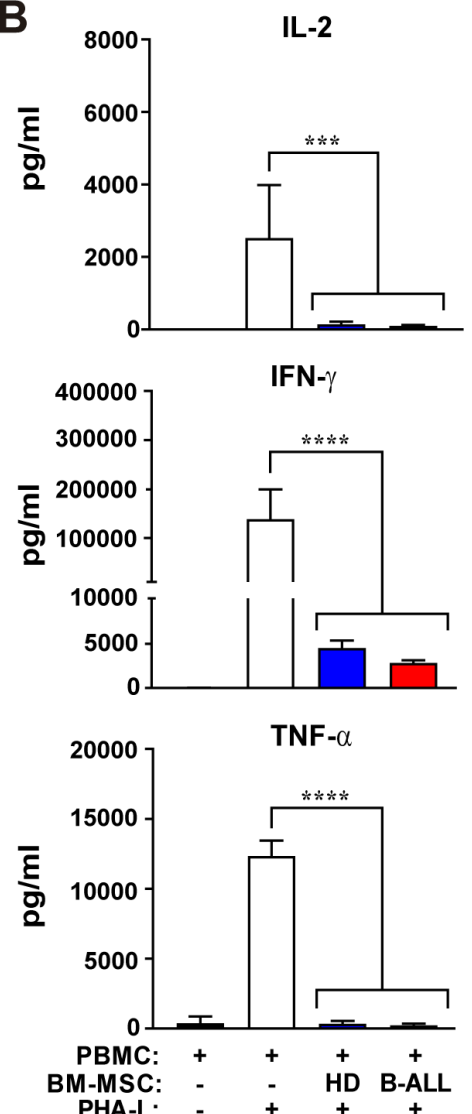

Figure 2 In vitro immunomodulatory properties of BM-MSC from pediatric patients with B-ALL and age-matched HD on T-cells. (A) Left panel, percentage of proliferating T-cells measured as percentage of CFSE ${ }^{+}$T-cells is shown. CellTrace CFSElabeled PBMC ( $n=3$ independent PBMC) was stimulated with PHA-L in the absence/presence of BM-MSC for 6 days at two different BM-MSC:PBMC ratios (1:5 and 1:10). Right panel, representative FACS histograms of CellTrace CFSE-labeled PBMC: R1 identifies non-proliferating CFSE ${ }^{++}$cells, and R2 identifies CFSE ${ }^{\text {low }}$ proliferating cells. (B) Secretion of the proinflammatory cytokines IL-2, IFN- $\gamma$ and TNF- $\alpha$ in cell-culture supernatants after 6 days at a BM-MSC:PBMC ratio of 1:10. Data are shown as mean \pm SEM. ${ }^{* \star \star} P<0.001,{ }^{* \star \star *} p<0.0001$; one-way ANOVA with Tukey's post hoc test. HD BM-MSC $n=3$ and B-ALL BM-MSC $n=5$. ANOVA, analysis of variance; B-ALL, B-cell acute lymphoblastic leukemia; BM-MSC, bone marrow-mesenchymal stem/stromal cells; HD, healthy donors; IFN- $\gamma$, interferon- $\gamma$; IL-2, interleukin-2; PBMC, peripheral blood mononuclear cell; TNF- $\alpha$, tumor necrosis factor $\alpha$.

of adipogenic (CEBP $\alpha, P P A R-\gamma)$ and osteogenic $(B M P 2$, RUNX2) differentiation (figure 1E).

\section{B-ALL BM-MSC immunosuppress T-cell response}

MSCs are widely recognized for their immunomodulatory potential, including the inhibition of allogenic T-cell proliferation and the production of proinflammatory cytokines. ${ }^{19}{ }^{20}$ We, therefore, monitored PHA-Lstimulated T-cell division in the absence or presence of BM-MSC in vitro. In line with published findings, ${ }^{32} 33$ we found that HD BM-MSC strongly inhibited T-cell proliferation in a dose-dependent manner (figure 2A). Of note, comparable inhibition of T-cell proliferation was exerted by B-ALL BM-MSC (figure 2A). We next analyzed these supernatants to test whether BM-MSC also regulate proinflammatory cytokine secretion. The analysis of supernatants showed that the levels of IL-2, IFN- $\gamma$ and TNF- $\alpha$ were comparably and significantly lower in $\mathrm{HD}$ and B-ALL BM-MSC cocultures than in PBMC-only controls (figure 2B). Overall, these results show that both HD and
B-ALL BM-MSC are equally immunosuppressive, as previously described. ${ }^{32} 33$

\section{B-ALL BM-MSC exert anti-inflammatory effects in a preclinical model of severe acute colitis}

Having confirmed the immunosuppressive properties of B-ALL BM-MSC in vitro, we wished to test their ability to influence T-cell functions in vivo. To do this, we used a wellestablished preclinical model of acute colitis (figure 3A) that shares clinical, histopathological and immunological features with Crohn's disease. ${ }^{30} 31$ As expected, TNBStreated mice developed severe, acute illness characterized by substantial $(\sim 20 \%)$ and sustained body weight loss (figure 3B), bloody diarrhea, rectal prolapse and pancolitis, accompanied by extensive wasting syndrome (figure 3C), which caused 25\% mortality over a 9-day period (figure 3D). Macroscopic examination of colons revealed profound signs of inflammation, hyperemia, ulceration and shortening (figure 3E). By contrast, mice that were treated with either HD or B-ALL BM-MSC 
A

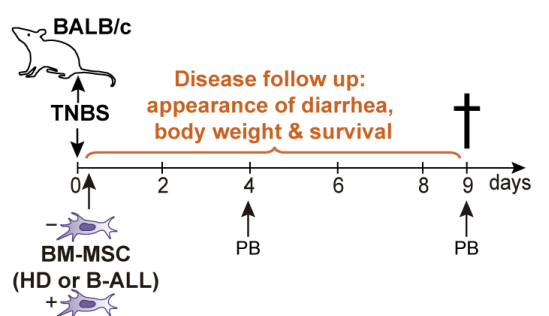
+2 or
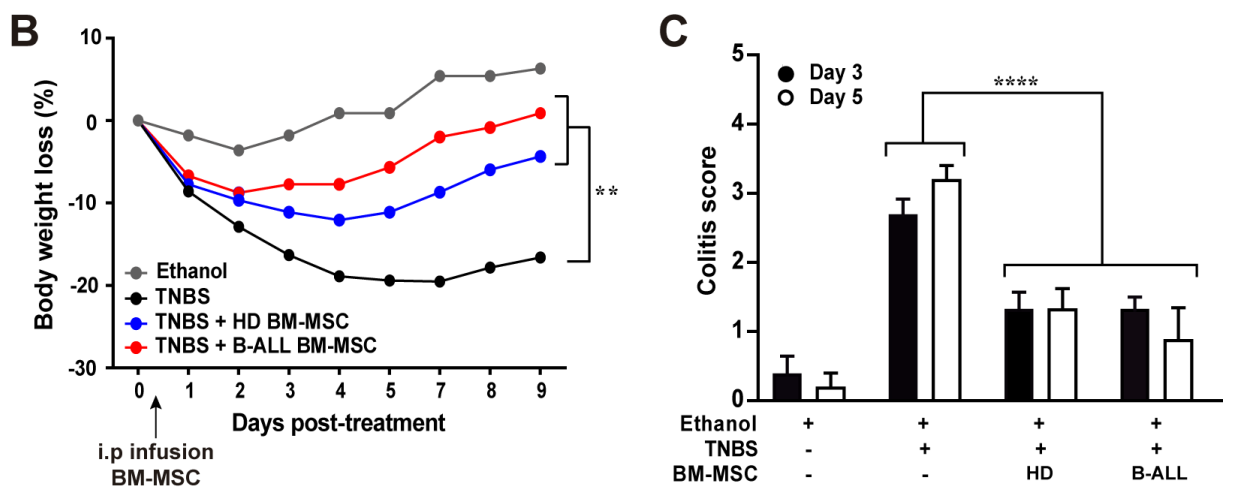

$\mathbf{E}$

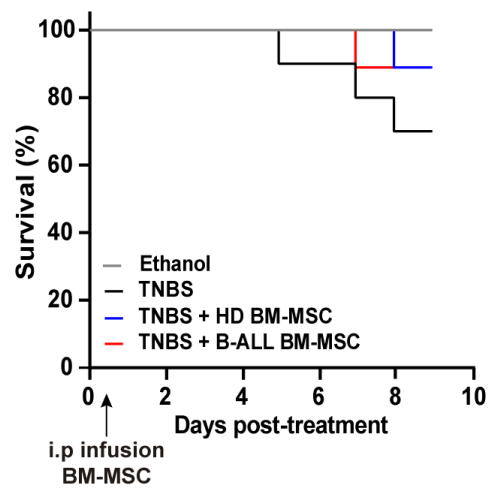

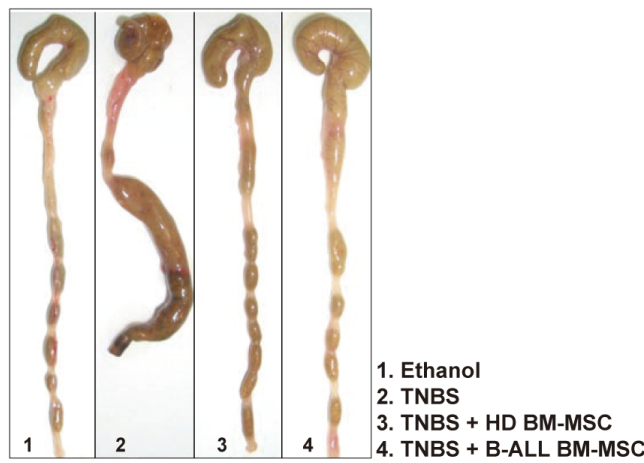

IL-6
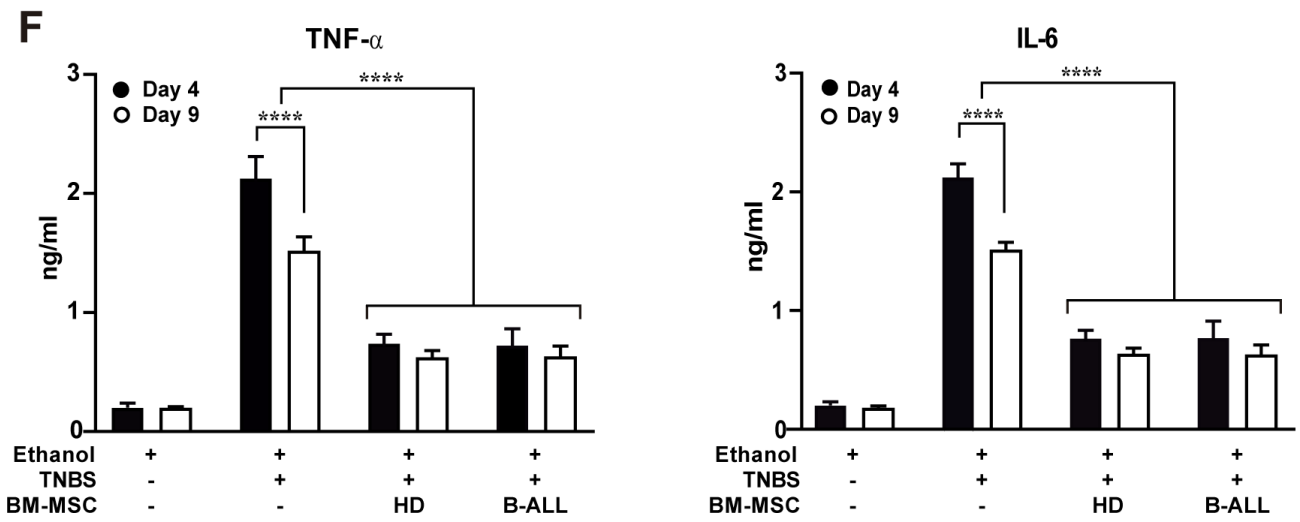

Figure 3 In vivo anti-inflammatory properties of BM-MSC from pediatric patients with B-ALL and HD in a model of acute colitis. (A) Scheme of the experimental design. Acute colitis was induced in mice by intrarectal administration of TNBS. After 4 hours, mice ( $n=5-10 /$ group) were injected intraperitoneally with medium or with HD or B-ALL BM-MSC. Body weight and survival were followed up for 9 days. (B) Daily change in body weight loss relative to day 0 (induction of colon damage). (C) Survival over a 9-day follow-up. (D) Colitis score determined at day 3 and 5. (E) Macroscopic damage score was evaluated in colons at sacrifice (day 9). (F) Serum levels of IL-6 and TNF- $\alpha$ at day 4 and 9. Data are shown as mean \pm SEM. ${ }^{* * *} P<0.001$, ${ }^{* * \star *} \mathrm{p}<0.0001$; one-way ANOVA test with Tukey's post hoc test. HD BM-MSC $n=3$ and B-ALL BM-MSC $n=3$. ANOVA, analysis of variance; B-ALL, B-cell acute lymphoblastic leukemia; BM-MSC, bone marrow-mesenchymal stem/stromal cells; HD, healthy donors; IL-6, interleukin-6; TNBS, 2,4,6-trinitrobenzene sulfonic acid; TNF- $\alpha$, tumor necrosis factor $\alpha$.

were largely protected from colitis, as evidenced by a significant recovery of their body weight loss within 9 days (figure 3B), and by the improvement in the wasting syndrome and the signs of colon inflammation, which was reflected in the significantly lower mortality and colitis score as compared with control animals (figure 3C-E). In accord with their anti-inflammatory effects, treatment with either HD or B-ALL BM-MSC decreased the levels of the proinflammatory cytokines TNF- $\alpha$ and IL- 6 in the sera of colitic mice (figure 3F). Collectively, our findings show that B-ALL BM-MSC can suppress inflammation in vivo to significantly protect mice against severe acute colitis.

\section{BM-MSC do not compromise CD19-CAR T-cell activity in vitro and in vivo}

While it is well established the contribution of BM-MSC in promoting survival, proliferation and chemotherapy resistance in B-ALL ${ }^{8-14}$ (online supplementary figure S2), the potential impact of B-ALL BM-MSC on CD19-CAR T-cell therapy has not been explored. Moreover, CAR 
A

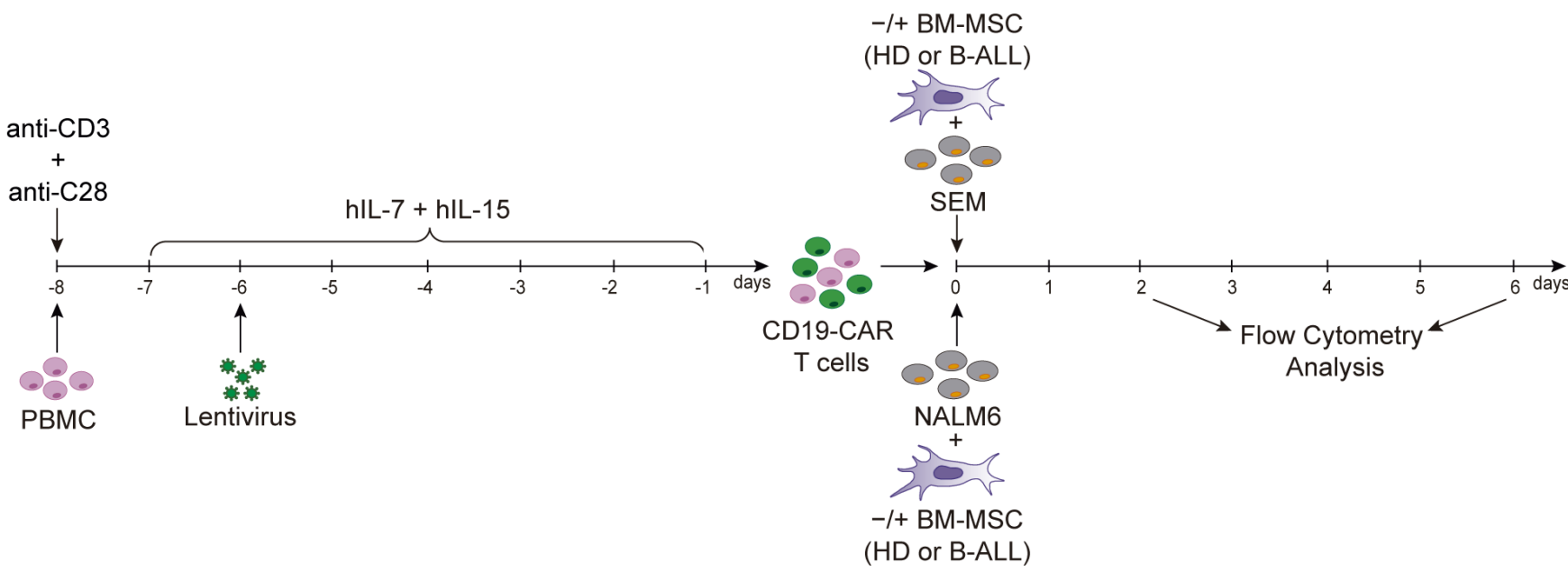

B
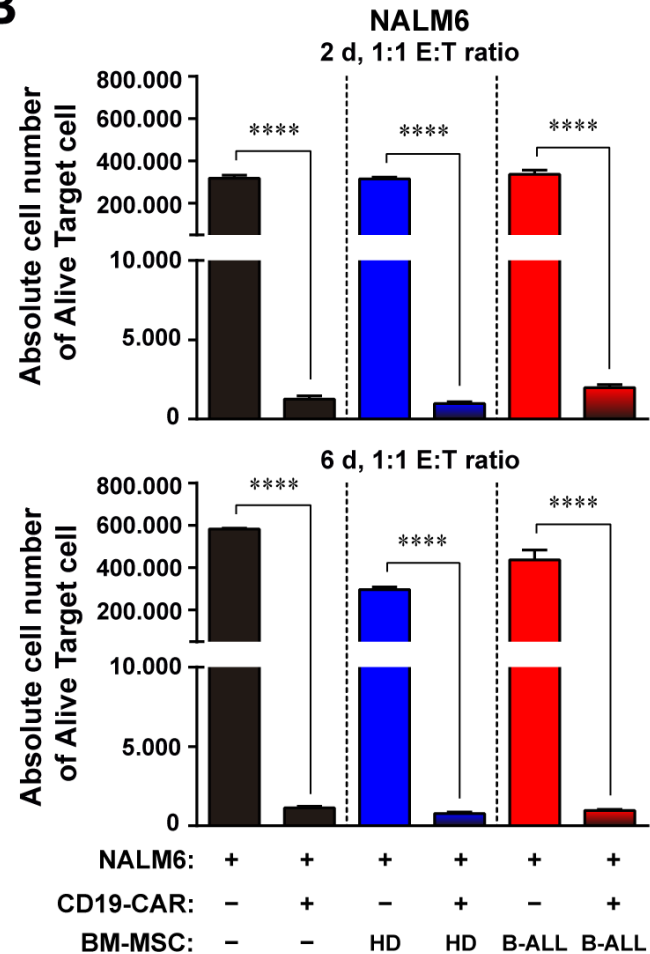

C

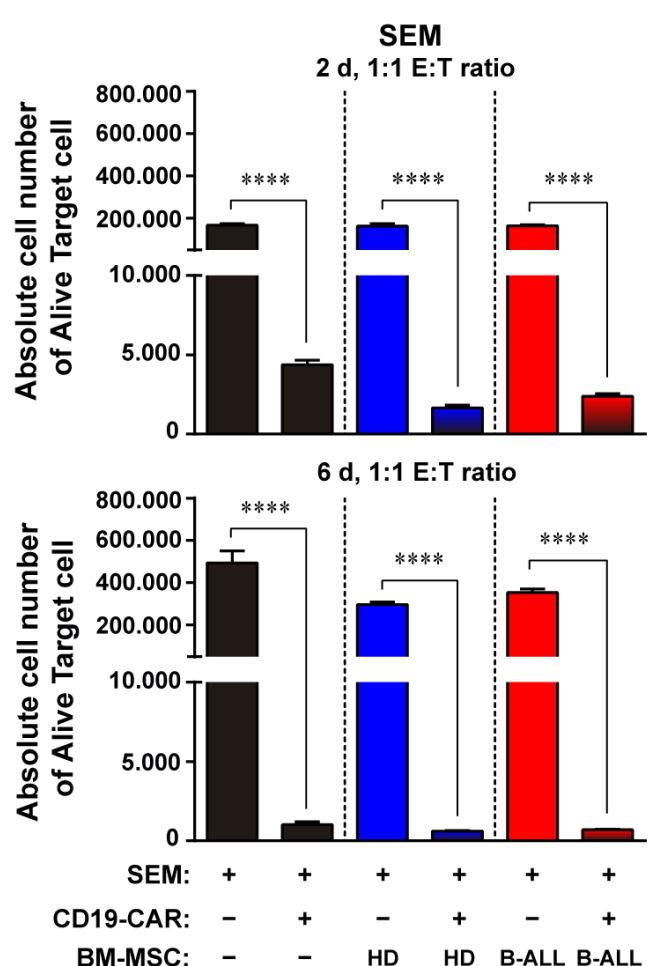

Figure 4 In vitro cytotoxicity of CD19-CAR T-cells against B-ALL cell lines in the absence/presence of BM-MSC. (A) Achematic of the experimental design for assessing the cytotoxicity of CD19-CAR T-cells against NALM6 or SEM B-ALL cell lines in the absence or presence of BM-MSC. (B, C) Absolute counts of alive target cells NALM6 (B) and SEM (C) measured by FACS after 2 and 6 days at 1:1 E:T ratio in the absence/presence of BM-MSC. Data are shown as mean \pm SEM. ${ }^{* \star \star *} P<0.0001$; one-way ANOVA test with Tukey's post hoc test. HD BM-MSC $n=3$ and B-ALL BM-MSC $n=5$. ANOVA, analysis of variance; B-ALL, Bcell acute lymphoblastic leukemia; BM-MSC, bone marrow-mesenchymal stem/stromal cells; E:T, Effector:Target; HD, healthy donors.

T-cells are likely to experience the same loss of function/ persistence as T-cells in an immunosuppressive B-ALL BMM. Consequently, we next explored the influence of BM-MSC on CD19-CAR T-cell activity. We first evaluated in vitro the impact of HD and B-ALL BM-MSC on CD19-CAR T-cell performance. We generated CD19-CAR T-cells following a standard protocol with anti-CD3/antiCD28 plus hIL-7 and hIL- $15,{ }^{29}$ and studied the effects of BM-MSC on CD19-CAR T-cell cytotoxicity against NALM6 and SEM cells cultured at a 1:1 E:T ratio for 2 and 6 days (figure 4A). We found that the number of CD19-CARresistant NALM6 or SEM cells cultured in the presence of either HD or B-ALL BM-MSC was very similar to that of target cells exposed to CD19-CAR T-cells alone, a non-protective role of BM-MSC on NALM6 (figure 4B) and SEM (figure 4C) cell survival in CD19-CAR T-cell therapy, at least in vitro. We additionally studied the ex vivo impact of B-ALL BM-MSC on CD19 CAR T-cell cytotoxicity against autologous primary B-ALL blasts, which partially replicates the pathophysiological conditions of 
A

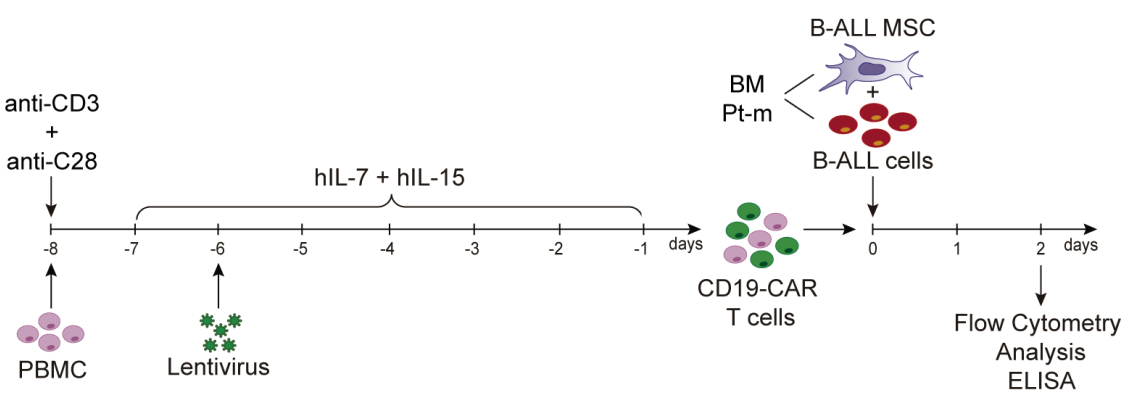

B

2d, 1:1 E:T ratio
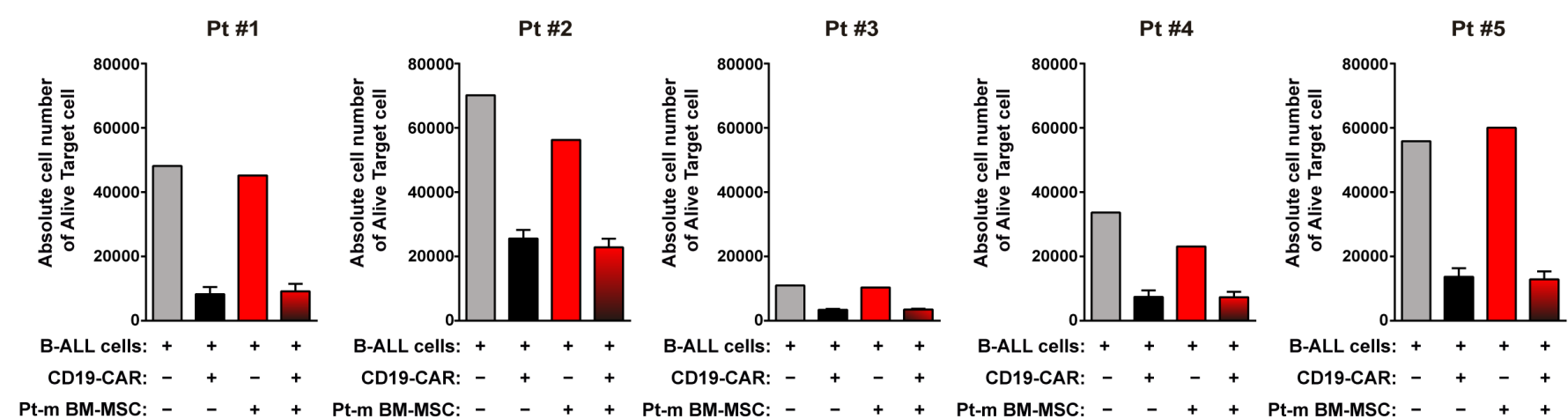

C
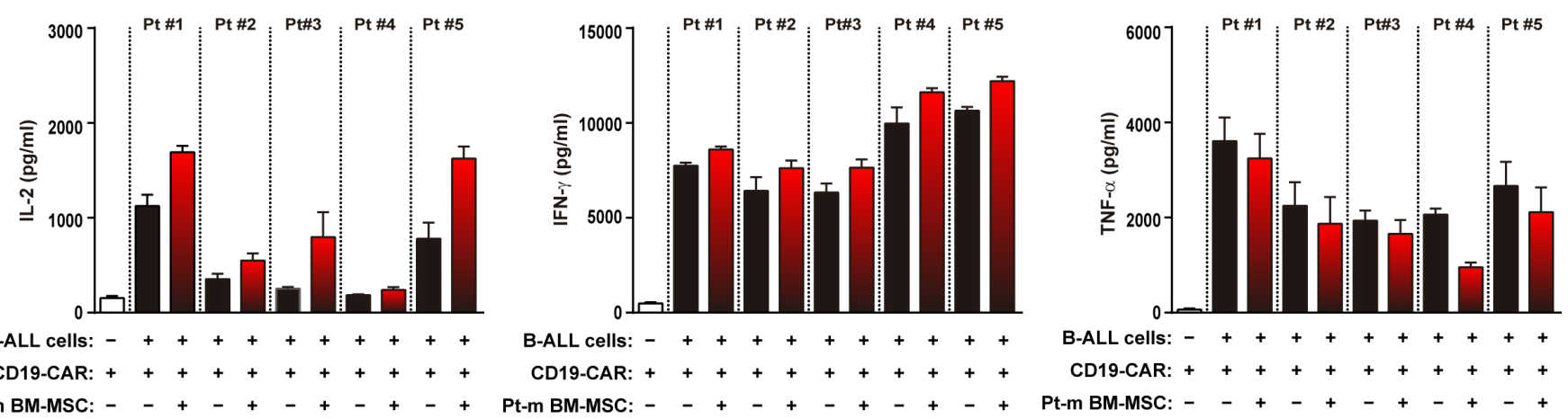

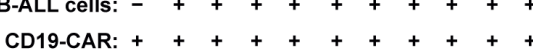

Figure 5 In vitro cytotoxicity of CD19-CAR T-cells against primary B-ALL blasts in the presence/absence of patient-matched BM-MSC. (A) Schematic of the experimental design for assessing the cytotoxicity of CD19-CAR T-cells against primary B-

ALL blasts in the absence or presence of patient-matched (Pt-m) BM-MSC. (B) Absolute counts of alive primary B-ALL blasts measured by FACS after 2 days cytotoxicity assay at a 1:1 E:T ratio in the absence/presence of Pt-m BM-MSC. (C) Secretion of IL-2, IFN- $\gamma$ and TNF- $\alpha$ by CD19-CAR T-cells ( $n=3$ independent PBMC) after 2 days exposure to primary B-ALL cells at a 1:1 E:T ratio in the absence/presence of Pt-m BM-MSC. Data are shown as mean \pm SEM B-ALL BM-MSC $n=5$. B-ALL, B-cell acute lymphoblastic leukemia; BM-MSC, bone marrow-mesenchymal stem/stromal cells; CAR, chimeric antigen receptor; E:T, Effector:Target; IFN- $\gamma$, interferon- $\gamma$; IL-2, interleukin-2; PBMC, peripheral blood mononuclear cell; TNF- $\alpha$, tumor necrosis factor $\alpha$.

patients. We performed cytotoxicity assays using B-ALL blasts and patient-matched derived BM-MSC from five pediatric B-ALL patients (figure 5A). Irrespective of the difference in survival of B-ALL primary cells, after 2 days of culture the number of alive/resistant primary blasts from the five patients after CD19-CAR T-cell treatment was very similar in the absence or presence of autologous BM-MSC (figure 5B), consistent with the results obtained using B-ALL cell lines (figure 4). In addition, we found no significant differences in the levels of IL-2, IFN- $\gamma$ and TNF- $\alpha$ in culture supernatants (figure $5 \mathrm{C}$ ), confirming that BM-MSC from pediatric B-ALL do not promote resistance to CD19-CAR T-cells in vitro.
Finally, we evaluated the impact of HD or B-ALL BM-MSC on CD19 CAR T-cell cytotoxicity in vivo, using a mouse xenograft model with NALM6 cells. To recapitulate the interactions between the BM-MSC and leukemic cells, $1 \times 10^{6}$ NALM6-Luc ${ }^{+}$cells were IT injected into NSG mice alone or together with $3 \times 10^{5} \mathrm{HD}$ or B-ALL BM-MSC, followed 4 days later by $5 \times 10^{6}$ CAR-T cells (figure $6 \mathrm{~A}$ ). Mice were followed up weekly by BLI. CD19-CAR T-cell-untreated mice showed massive leukemic burden and succumbed quickly to the disease (figure $6 \mathrm{~B}$ and $\mathrm{C}$ ). In contrast, mice treated with CD19-CAR T-cells, irrespective of whether they were transplanted or not with BM-MSC, similarly controlled the disease (figure 6B and $\mathrm{C}$ ). FACS analysis of leukemic burden 
A

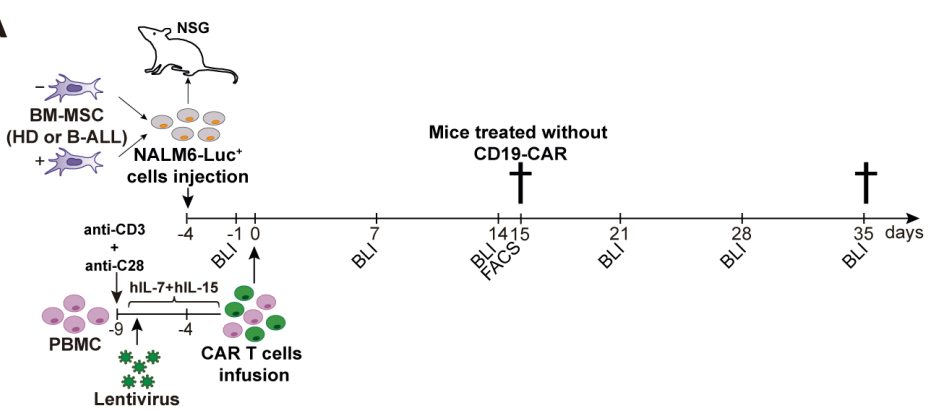

B

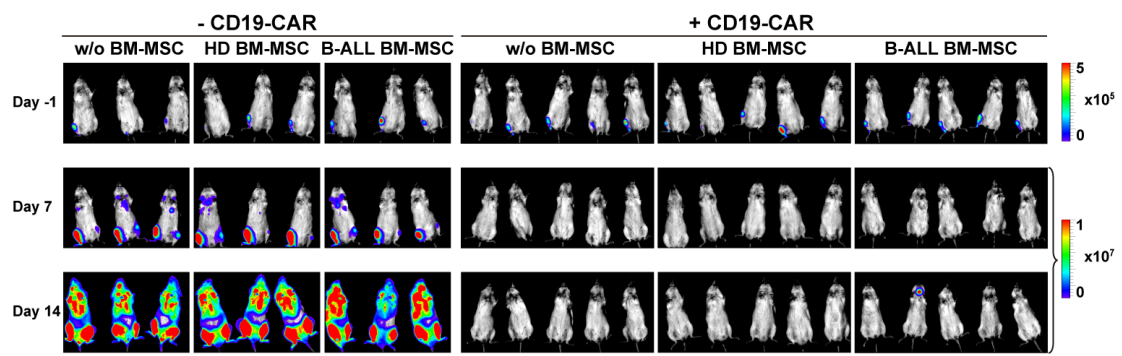

C

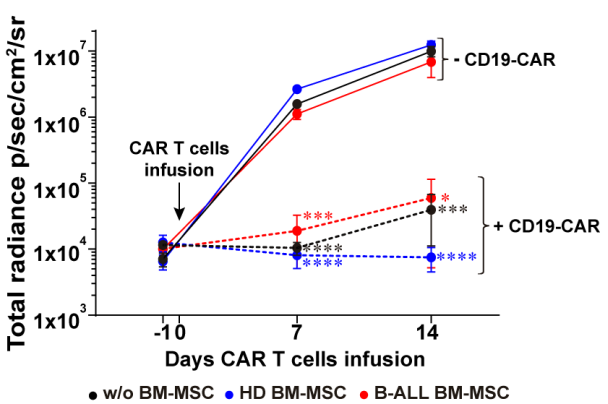

D

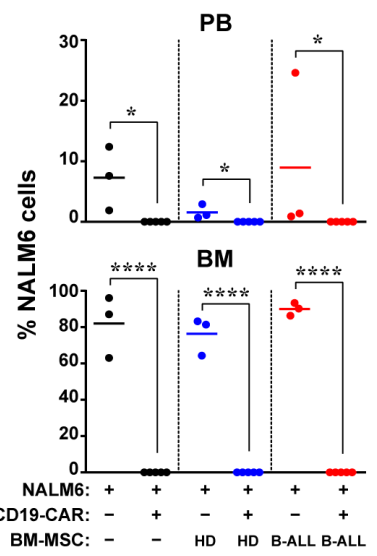

E

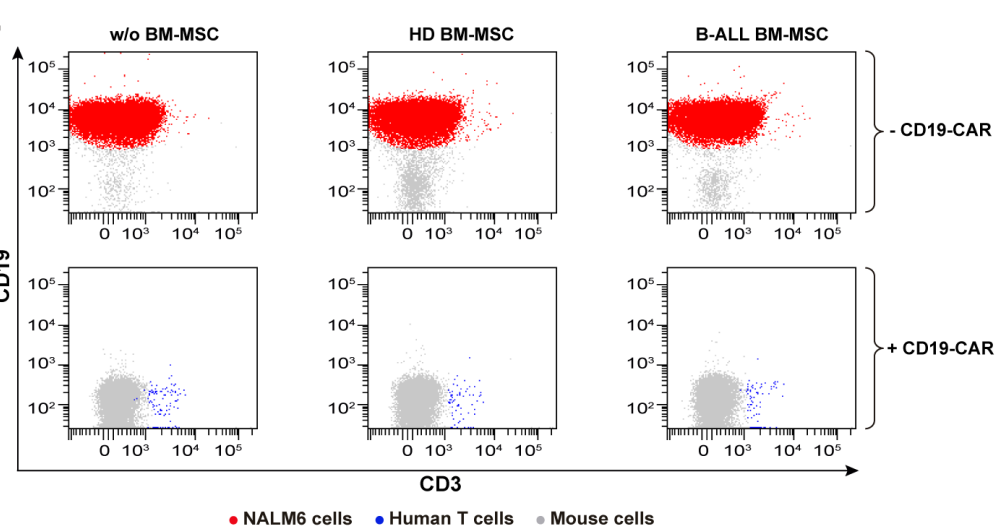

$\mathbf{F}$

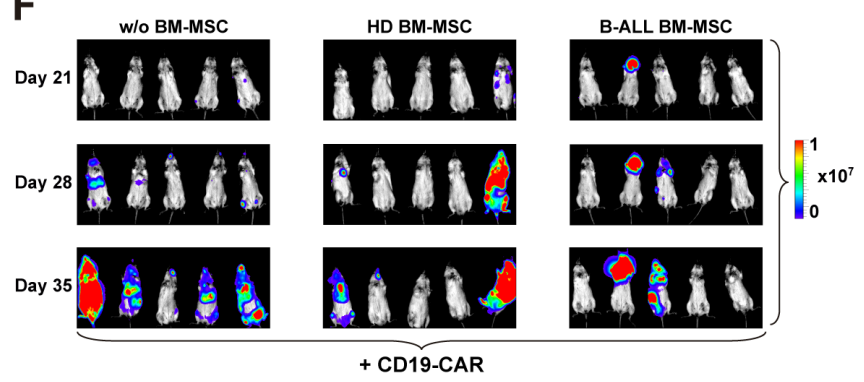

G

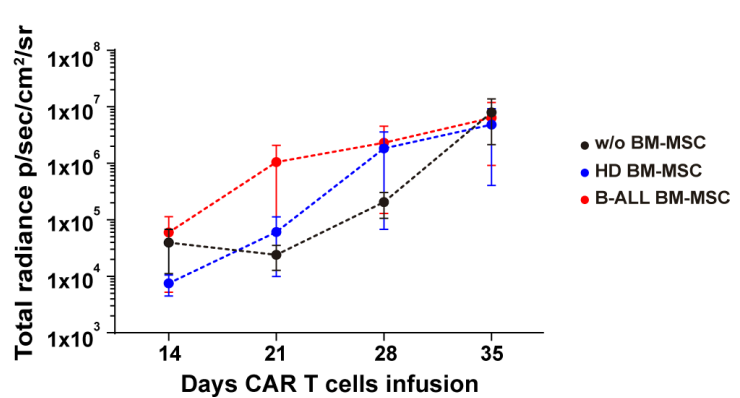

Figure 6 In vivo cytotoxicity of CD19-CAR T-cells in an NALM6 mouse xenograft model in the absence/presence of BM-MSC. (A) Schematic of the transplantation of B-ALL cells and BM-MSC followed by CD19-CAR T-cell infusion after 4 days to assess in vivo the influence of BM-MSC on CD19-CAR T-cell activity. (B) Leukemic burden monitored weekly (until sacrifice of control mice) by BLI. (C) Total RADIANCE quantification ( $\mathrm{p} / \mathrm{sec} / \mathrm{cm}^{2} / \mathrm{sr}$ ) of mice shown in B. (D) Leukemic burden analyzed by FACS in PB and BM at the time control mice were sacrificed (day 15). (E) Representative FACS dot plots identifying target cells (red) and T-cell persistence (blue) in BM at the time control mice were sacrificed. (F) Long-term (35 days) BLI follow-up of CD19-CAR Tcell-treated mice in the absence/presence of BM-MSC. (G) Total RADIANCE quantification (p/sec/ $\mathrm{cm}^{2} / \mathrm{sr}$ ) of mice shown in F). Each dot represents a mouse. Data are shown as mean \pm SEM. ${ }^{*} \mathrm{P}<0.05,{ }^{* * *} \mathrm{p}<0.001,{ }^{* * *} \mathrm{p}<0.0001$; a paired two-tailed t-test was used when two experimental groups were compared, and one-way ANOVA test with Tukey's post hoc test was used when three experimental groups were compared. ANOVA, analysis of variance; B-ALL, B-cell acute lymphoblastic leukemia; BM-MSC, bone marrow-mesenchymal stem/stromal cells; CAR, chimeric antigen receptor; E:T, Effector:Target; PB, peripheral blood. 
in $\mathrm{PB}$ and BM at day 15 confirmed the BLI data (figure 6D and E). All those mice treated with CD19-CAR T-cells with or without HD/B-ALL BM-MSCs in which the disease was controlled at the time of sacrificing the untreated controls (day 15) were followed up to 35 days, and found that neither HD nor B-ALL BM-MSC impaired long-term CD19-CAR T-cell activity (figure 6F,G). Collectively, neither HD nor B-ALL BM-MSCs apparently contribute to CD19-CAR T-cell resistance.

\section{DISCUSSION}

There is increasing evidence that the BMM can influence leukemia progression. ${ }^{34-37}$ In this context, BM-MSCs are considered important players in the BM, promoting proliferation, survival, migration, vascularization and chemoresistance, and suppressing immune cells, all key processes for leukemia progression and immune evasion. ${ }^{19} 203637$ B-ALL is malignant proliferation of $\mathrm{CD} 19^{+} \mathrm{B}$-cell precursors, ${ }^{1}$ and BM-MSCs have been shown to promote B-ALL progression and chemoresistance by several mechanisms. ${ }^{4-14} 3839$ Here, we obtained BM-MSC from pediatric patients with B-ALL and compared their functional and immunological characteristics with those of BM-MSC from age-matched HD. We show that both HD and B-ALL BM-MSC display comparable morphology, immunophenotype and differentiation potential in vitro; however, B-ALL BM-MSCs are less proliferative. This is in line with defects in B-ALL BM-MSC expansion reported by others. ${ }^{32}{ }^{33}$ In addition, we show that B-ALL and HD BM-MSC suppress mitogenic T-cell proliferation by PHA-L in a dose-dependent manner, and block the secretion of pro-inflammatory cytokines by T-cells in coculture. Overall, these data indicate that the typical in vitro biological properties of BM-MSC, including their immunosuppressive effects, are unchanged in patients with B-ALL, which is in agreement with earlier reports. ${ }^{32} 33$

The in vivo anti-inflammatory properties of BM-MSC from patients with hematological malignancies have been scarcely investigated. ${ }^{40}$ In the present study, we describe, for the first time to our knowledge, the in vivo anti-inflammatory effects of BM-MSC from pediatric patients with B-ALL using a preclinical model of acute colitis. TNBS-treated mice develop a severe acute illness while mice treated with $\mathrm{HD}$ or B-ALL BM-MSC are protected against colitis. Also, the levels of master pro-inflammatory cytokines in the serum of colitis mice treated with HD or B-ALL BM-MSC are diminished. Collectively, our results show that HD and B-ALL BM-MSC have a similar capacity to dampen inflammation in a wellcharacterized in vivo model.

Given the accumulating evidence that BM-MSCs protect B-ALL cells from chemotherapy in vitro, ${ }^{8-14}$ we confirmed that coculturing B-ALL BM-MSC with B-ALL cells reduces apoptosis in leukemic cells in response to dexamethasone and L-asparaginase, with HD BM-MSC showing a similar capacity to protect leukemic cells from chemotherapy. Whether B-ALL MSC could protect B-ALL cells during CD19-CAR T-cell therapy was, however, not known. Thus, we evaluated whether BM-MSC participated in the resistance of B-ALL to CD19-CAR T-cell therapy. We found that CD19-CAR T-cells can efficiently eliminate both B-ALL cell lines and B-ALL primary cells in vitro regardless the absence/presence of either HD or B-ALL BM-MSC. Moreover, when we analyzed the proinflammatory cytokines secreted in the cocultures of B-ALL blasts with patientmatched BM-MSC derived from pediatric B-ALL patients we found no significant differences as compared with those in the absence of BM-MSC, suggesting that BM-MSC from pediatric B-ALL patients do not compromise CD19-CAR T-cell efficacy in vitro. These findings concur with a previous study by Campana and colleagues. ${ }^{41}$ We also provide the first demonstration, to the best of our knowledge, that in vivo HD or B-ALL BM-MSC do not contribute to CD19-CAR T-cell resistance using a mouse xenograft model which recapitulates the interactions between the BM-MSC and B-ALL cells.

In conclusion, our data show that pediatric B-ALL BM-MSC and age-matched HD BM-MSC equally immunosuppressive T-cell response, but do not compromise CD19-CAR T-cell activity either in vitro or in vivo, at least within the experimental constraints of this study. Although this study indicates that specific immunobiological properties of BM-MSCs do not seem to underlie the resistance of B-ALL cells to CD19-CAR T-cells, we must bear in mind that our study does not unequivocally assess the contribution of the intact BMM, composed of different cell types and cellular interactions, to CD19-CAR T-cells mediated cytotoxicity. Further in vivo studies using humanized animal models are necessary to elucidate the impact of the BMM on the response to adoptive cell therapy with CD19-CAR T-cells.

\section{Author affiliations}

${ }^{1}$ Josep Carreras Leukemia Research Institute, Barcelona, Spain

${ }^{2}$ Servicio de Inmunología, Hospital Clínico de Barcelona, Hospital Clínico de Barcelona, Barcelona, Spain

${ }^{3}$ Sección de Oncohematología Pediátrica, Hospital Clínico Universitario Virgen de la Arrixaca, El Palmar, Murcia, Spain

${ }^{4}$ Instituto Murciano de Investigación biosanitaria, Murcia, Spain

${ }^{5}$ Hematology Laboratory, Hospital Sant Joan de Déu, University of Barcelona, Barcelona, Spain

${ }^{6}$ Institut de Recerca Hospital Sant Joan de Déu Barcelona, Barcelona, Spain ${ }^{7}$ Centro de Investigación Biomédica en Red de Enfermedades Raras (CIBERER),

ISCIII, Barcelona, Spain

${ }^{8}$ Blood and Tissue Bank, Barcelona, Spain

${ }^{9}$ Instituto de Parasitología y Biomedicina López-Neyra-CSIC, Barcelona, Spain

${ }^{10}$ Institució Catalana de Recerca i Estudis Avançats, Barcelona, Spain

${ }^{11}$ Centro de Investigación Biomédica en Red de Cáncer (CIBERONC), ISCIII, Barcelona, Spain

Acknowledgements We are indebted to our laboratory colleagues and especially to Ana Belén Carrillo (GENYO, Granada, Spain) y Rosa Maria Yañez (CIEMAT, Madrid, Spain) for their technical feedback and constructive discussions. We thank CERCA/ Generalitat de Catalunya and Fundació Josep Carreras-Obra Social la Caixa for their institutional support.

Contributors SRZ conceived the study, designed and performed experiments, analyzed data, interpreted data, performed figures and wrote the manuscript. PAR designed and performed experiments, analyzed data, interpreted data, performed figures and reviewed the paper. MV performed experiments. JLF, MC, $\mathrm{SQ}$ and MJ provided human samples. MD performed experiments, analyzed data 
and interpreted data. PM, conceived the study, designed experiments, wrote the manuscript, and financially supported the study.

Funding Financial support for this work was obtained from the European Research Council (CoG-2014-646903, PoC-2018-811220), the Spanish Ministry of Economy and Competitiveness (MINECO, SAF2016-80481R), the Fundación Uno entre Cienmil, the Obra Social La Caixa (LCF/PR/HR19/52160011), the Leo Messi Foundation, and the 'Heroes hasta la médula' initiative to PM. SRZ was supported by a Marie Sklodowska Curie Fellowship (GA 795833). MV is supported by a Juan de la Cierva fellowship from the MINECO. PM is an investigator of the Spanish Cell Therapy cooperative network (TERCEL).

Competing interests None declared.

Patient consent for publication Not required.

Ethics approval The study was approved by the institutional review board of the Ethics Committee on Clinical Research of Clinic Hospital of Barcelona (HCB/2017/0781). Animal studies of experimental acute severe colitis were conducted with the approval of the Ethics Committee of the Spanish Council of Scientific Research and in vivo CD19-CAR T cell-mediated cytotoxicity assay was approved by the Barcelona Biomedical Research Park ethics committee (HRH-170029-P1). The in vivo procedure was approved by the local ethics committee ( $\mathrm{HRH}-$ 17-0029 P1).

Provenance and peer review Not commissioned; externally peer reviewed.

Data availability statement Data are available on reasonable request. All data relevant to the study are included in the article or uploaded as online supplementary information. Data are available on reasonable request. All data relevant to the study are included in the article or uploaded as online supplementary information.

Open access This is an open access article distributed in accordance with the Creative Commons Attribution Non Commercial (CC BY-NC 4.0) license, which permits others to distribute, remix, adapt, build upon this work non-commercially, and license their derivative works on different terms, provided the original work is properly cited, appropriate credit is given, any changes made indicated, and the use is non-commercial. See http://creativecommons.org/licenses/by-nc/4.0/.

\section{ORCID iD}

Samanta Romina Zanetti http://orcid.org/0000-0002-9052-8751

\section{REFERENCES}

1 Katz AJ, Chia VM, Schoonen WM, et al. Acute lymphoblastic leukemia: an assessment of international incidence, survival, and disease burden. Cancer Causes Control 2015;26:1627-42.

2 Winters AC, Bernt KM. MII-Rearranged Leukemias-An update on science and clinical approaches. Front Pediatr 2017;5:4

3 Pui CH, Crist WM. Biology and treatment of acute lymphoblastic leukemia. J Pediatr 1994;124:491-503.

4 Gibson LF. Survival of B lineage leukemic cells: signals from the bone marrow microenvironment. Leuk Lymphoma 2002;43:19-27.

5 Bradstock KF, Gottlieb DJ. Interaction of acute leukemia cells with the bone marrow microenvironment: implications for control of minimal residual disease. Leuk Lymphoma 1995;18:1-16.

6 LeClerc JM, Billett AL, Gelber RD, et al. Treatment of childhood acute lymphoblastic leukemia: results of Dana-Farber all Consortium protocol 87-01. J Clin Oncol 2002;20:237-46.

7 Hoogerbrugge PM, Gerritsen EJ, vd Does-van den Berg A, et al. Case-Control analysis of allogeneic bone marrow transplantation versus maintenance chemotherapy for relapsed all in children. Bone Marrow Transplant 1995;15:255-9.

8 Burt R, Dey A, Aref S, et al. Activated stromal cells transfer mitochondria to rescue acute lymphoblastic leukemia cells from oxidative stress. Blood 2019;134:1415-29.

9 Moses BS, Slone WL, Thomas P, et al. Bone marrow microenvironment modulation of acute lymphoblastic leukemia phenotype. Exp Hematol 2016;44:50-9.

10 Mudry RE, Fortney JE, York T, et al. Stromal cells regulate survival of B-lineage leukemic cells during chemotherapy. Blood 2000;96:1926-32.

11 Randhawa S, Cho BS, Ghosh D, et al. Effects of pharmacological and genetic disruption of CXCR4 chemokine receptor function in Bcell acute lymphoblastic leukaemia. Br J Haematol 2016;174:425-36.

12 Tesfai Y, Ford J, Carter KW, et al. Interactions between acute lymphoblastic leukemia and bone marrow stromal cells influence response to therapy. Leuk Res 2012;36:299-306.

13 Yang Y, Mallampati S, Sun B, et al. Wnt pathway contributes to the protection by bone marrow stromal cells of acute lymphoblastic leukemia cells and is a potential therapeutic target. Cancer Lett 2013;333:9-17.

14 Yu K, Yin Y, Ma D, et al. Shp2 activation in bone marrow microenvironment mediates the drug resistance of B-cell acute lymphoblastic leukemia through enhancing the role of VCAM-1/NLA4. Int Immunopharmacol 2020;80:106008.

15 Rafei H, Kantarjian HM, Jabbour EJ. Recent advances in the treatment of acute lymphoblastic leukemia. Leuk Lymphoma 2019;60:2606-21.

16 Sermer D, Brentjens R. Car T-cell therapy: full speed ahead. Hematol Oncol 2019;37:95-100.

17 Ruella M, Maus MV. Catch me if you can: leukemia escape after CD19-Directed T cell immunotherapies. Comput Struct Biotechnol J 2016;14:357-62.

18 Gardner R, Wu D, Cherian S, et al. Acquisition of a CD19-negative myeloid phenotype allows immune escape of MLL-rearranged B-ALL from CD19 CAR-T-cell therapy. Blood 2016;127:2406-10.

19 Guerrouahen BS, Sidahmed H, Al Sulaiti A, et al. Enhancing mesenchymal stromal cell immunomodulation for treating conditions influenced by the immune system. Stem Cells Int 2019;2019:1-11.

20 Regmi S, Pathak S, Kim JO, et al. Mesenchymal stem cell therapy for the treatment of inflammatory diseases: challenges, opportunities, and future perspectives. Eur J Cell Biol 2019;98:151041.

21 Zanetti SR, Ziblat A, Torres NI, et al. Expression and functional role of $\alpha 7$ nicotinic receptor in human cytokine-stimulated natural killer (NK) cells. J Biol Chem 2016;291:16541-52.

22 Diaz de la Guardia R, Lopez-Millan B, Lavoie JR, et al. Detailed characterization of mesenchymal stem/stromal cells from a large cohort of AML patients demonstrates a definitive link to treatment outcomes. Stem Cell Reports 2017;8:1573-86.

23 Lechanteur C, Briquet A, Giet O, et al. Clinical-scale expansion of mesenchymal stromal cells: a large banking experience. J Trans/ Med 2016;14:145.

24 Viswanathan S, Shi Y, Galipeau J, et al. Mesenchymal stem versus stromal cells: International Society for Cell \& Gene Therapy (ISCT®) Mesenchymal Stromal Cell committee position statement on nomenclature. Cytotherapy 2019;21:1019-24.

$25 \mathrm{Xu} \mathrm{X,} \mathrm{Li} \mathrm{R,} \mathrm{Zhou} \mathrm{Y,} \mathrm{et} \mathrm{al.} \mathrm{Dysregulated} \mathrm{systemic} \mathrm{lymphocytes}$ affect the balance of osteogenic/adipogenic differentiation of bone mesenchymal stem cells after local irradiation. Stem Cell Res Ther 2017;8:71.

26 Borge M, Nannini PR, Morande PE, et al. Cxcl12 is a costimulator for CD4+ T cell activation and proliferation in chronic lymphocytic leukemia patients. Cancer Immunol Immunother 2013;62:113-24.

27 Lopez-Millan B, Diaz de la Guardia R, Roca-Ho H, et al. Imids mobilize acute myeloid leukemia blasts to peripheral blood through downregulation of CXCR4 but fail to potentiate AraC/ Idarubicin activity in preclinical models of non del5q/5q- AML. Oncoimmunology 2018;7:e1477460.

28 Castella M, Boronat A, Martín-lbáñez R, et al. Development of a novel anti-CD19 chimeric antigen receptor: a paradigm for an affordable CAR T cell production at academic institutions. Mol Ther Methods Clin Dev 2019;12:134-44.

29 Baroni ML, Sanchez Martinez D, Gutierrez Aguera F, et al. 41BB-based and CD28-based CD123-redirected T-cells ablate human normal hematopoiesis in vivo. $\mathrm{J}$ Immunother Cancer 2020;8:e000845.

30 Gonzalez-Rey E, Anderson P, González MA, et al. Human adult stem cells derived from adipose tissue protect against experimental colitis and sepsis. Gut 2009;58:929-39.

31 Mizoguchi A, Mizoguchi E. Animal models of IBD: linkage to human disease. Curr Opin Pharmacol 2010;10:578-87.

32 Conforti A, Biagini S, Del Bufalo F, et al. Biological, functional and genetic characterization of bone marrow-derived mesenchymal stromal cells from pediatric patients affected by acute lymphoblastic leukemia. PLoS One 2013;8:e76989.

33 Zhi-Gang Z, Wei-Ming L, Zhi-Chao C, et al. Immunosuppressive properties of mesenchymal stem cells derived from bone marrow of patient with hematological malignant diseases. Leuk Lymphoma 2008;49:2187-95.

34 Ayala F, Dewar R, Kieran M, et al. Contribution of bone microenvironment to leukemogenesis and leukemia progression. Leukemia 2009;23:2233-41.

35 Méndez-Ferrer S, Bonnet D, Steensma DP, et al. Bone marrow niches in haematological malignancies. Nat Rev Cancer 2020;20:285-98.

36 Reagan MR, Rosen CJ. Navigating the bone marrow niche: translational insights and cancer-driven dysfunction. Nat Rev Rheumatol 2016;12:154-68.

37 Schepers K, Campbell TB, Passegué E. Normal and leukemic stem cell niches: insights and therapeutic opportunities. Cell Stem Cell 2015;16:254-67. 
38 Ma Z, Zhao X, Deng M, et al. Bone marrow mesenchymal stromal cell-derived periostin promotes B-ALL progression by modulating CCL2 in leukemia cells. Cell Rep 2019;26:1533-43.

39 Verma D, Zanetti C, Godavarthy PS, et al. Bone marrow niche-derived extracellular matrix-degrading enzymes influence the progression of B-cell acute lymphoblastic leukemia. Leukemia 2020;34:1540-52.

40 de la Guardia RD, Lopez-Millan B, Roca-Ho H, et al. Bone marrow mesenchymal stem/stromal cells from risk-stratified acute myeloid leukemia patients are anti-inflammatory in in vivo preclinical models of hematopoietic reconstitution and severe colitis. Haematologica 2019;104:e54-8.

41 Imai $\mathrm{C}$, Mihara K, Andreansky $\mathrm{M}$, et al. Chimeric receptors with 4-1BB signaling capacity provoke potent cytotoxicity against acute lymphoblastic leukemia. Leukemia 2004;18:676-84 